\title{
Short-term effects of transcatheter arterial chemoembolisation on metabolic activity of the liver of cirrhotic patients with hepatocellular carcinoma
}

S Bianco, C Merkel, S Savastano, S Bellon, M Chiesura-Corona, M Bolognesi, D Miotto, E Enzo, G Feltrin, A Gatta

\begin{abstract}
Background-Transcatheter arterial chemoembolisation, a procedure for the treatment of hepatocellular carcinoma, provokes a pronounced but transient increase in hepatic cytolysis parameters. A definite evaluation of the impairment of liver function after this treatment, performed by adequate techniques, is still lacking.

Aims-To assess and quantify the impairment of liver metabolic activity after arterial chemoembolisation in patients with cirrhosis. The variations of hepatic vein pressure gradient provoked by this procedure were evaluated.

Patients-15 patients with cirrhosis (Child's class A and B) and hepatocellular carcinoma.
\end{abstract}

Methods-17 transcatheter arterial chemoembolisations with epirubicin, iodised oil, and gelfoam were performed; liver function was assessed before, the following day, and after seven days measuring galactose elimination capacity; aminopyrine breath test was also performed in six patients before the procedure and seven days after. In 10 patients intrinsic hepatic clearance of indocyanine green and hepatic vein pressure gradient were measured by hepatic vein catheterisation before and 30 minutes after chemoembolisation.

Results-Intrinsic hepatic clearance of indocyanine green decreased significantly from (mean (SEM)) 355 (140) $\mathrm{ml} / \mathrm{min}$ to $277(98) \mathrm{ml} / \mathrm{min}$ after the procedure $(p=$ $0 \cdot 0007)$. Galactose elimination capacity did not show significant changes, being $4.00(0.90) \mathrm{mg} / \mathrm{min} / \mathrm{kg}$ body weight at baseline, $4.20(0.90) \mathrm{mg} / \mathrm{min} / \mathrm{kg}$ body weight after one day, and $3.95(0.87) \mathrm{mg} / \mathrm{min} / \mathrm{kg}$ body weight seven days after chemoembolisation. Aminopyrine breath test was $2.31(1.09) \%$ and remained unchanged after treatment, being $2.39(2.04) \%$ at day 7. Baseline hepatic vein pressure gradient was $17.0(5.5) \mathrm{mm} \mathrm{Hg}$, and $14.4(3.7) \mathrm{mm}$ Hg 30 minutes after chemoembolisation $(\mathbf{p}=0.09)$.

Conclusions-A single transcatheter chemoembolisation in cirrhotic patients provokes an acute and transient decrease in intrinsic hepatic clearance of indocyanine green; no liver function impairment was detected by galactose elimination capacity and aminopyrine breath test one and seven days after the procedure. Therefore it can be considered a safe therapeutic tool for hepatocellular carcinoma in Child's class $A$ and $B$ cirrhotic patients.

(Gut 1996; 39: 325-329)

Keywords: cirrhosis, liver function test, hepatocellular carcinoma, transcatheter arterial chemoembolisation.

Transcatheter arterial chemoembolisation (TACE) is one of the main treatment options for patients with advanced cirrhosis and hepatocellular carcinoma (HCC). ${ }^{12}$ In nearly $90 \%$ of patients with HCC liver cirrhosis coexists, and this feature limits treatment options because of the risk of aggravating liver insufficiency. Therefore, in most clinical settings, TACE is only used in patients with Child's class $A$ and $B$ disease. On the basis of pronounced but transient increase of parameters of hepatic cytolysis and considering the doubtful benefit on survival, ${ }^{3}$ a deleterious effect of TACE has been suspected; however a definite evaluation of the severity of such damage is still lacking. The small number of reports that used adequate quantitative techniques have conflicting results. ${ }^{45}$

There is little information also on the effect of TACE in portal hypertension, as only a single report showing no relevant effect in portal hypertension has been published to date. ${ }^{6}$ However, an increase in portal hypertension could be hypothesised on the basis of recent findings ${ }^{6,7}$ and this can contribute to the deterioration in liver function in such subjects.

The aim of this study was to assess the effects of TACE on liver function investigated by quantitative techniques independent on liver perfusion, and on hepatic venous pressure gradient.

\section{Methods}

\section{Patients}

From January 1993 to November 1994, 17 TACE procedures were performed in 15 cirrhotic patients (10 males and five females) with HCC, admitted to the University Hospital of Padua. Age ranged 33-69 years (average 62). The diagnosis of cirrhosis was 
Main clinical and biochemical baseline data of patients

\begin{tabular}{|c|c|c|c|c|c|c|c|c|c|c|c|}
\hline Patient & $\begin{array}{l}\text { Age } \\
(y)\end{array}$ & $\begin{array}{l}\text { Child- } \\
\text { Pugh } \\
\text { score }\end{array}$ & $\begin{array}{l}\text { Neoplastic } \\
\text { nodules } \\
\text { (n) }\end{array}$ & Ascites & $\begin{array}{l}\text { Total } \\
\text { protein } \\
(\mathrm{g} / \mathrm{l})\end{array}$ & $\begin{array}{l}\text { Albumin } \\
(g / l)\end{array}$ & $\begin{array}{l}P T \\
(\%)\end{array}$ & $\begin{array}{l}\text { Total } \\
\text { bilirubin } \\
\text { ( } \mu \text { moln) }\end{array}$ & $\begin{array}{l}\text { Conjugated } \\
\text { bilirubin } \\
\text { ( } \mu \text { moll })\end{array}$ & $\begin{array}{l}A S T \\
\text { (Un) }\end{array}$ & $\begin{array}{l}A L T \\
(U /)\end{array}$ \\
\hline 1 & 52 & 7 & $\mathbf{M}$ & $\mathbf{N}$ & 82 & 45 & 76 & $35 \cdot 5$ & $8 \cdot 6$ & 43 & 32 \\
\hline 2 & 59 & 9 & $\mathbf{M}$ & $\mathbf{N}$ & 87 & 30 & 40 & $47 \cdot 2$ & $21 \cdot 4$ & 321 & 203 \\
\hline 3 & 67 & 7 & $S$ & $Y$ & 75 & 38 & 57 & $20 \cdot 1$ & $6 \cdot 4$ & 40 & 31 \\
\hline 4 & 67 & 6 & $\mathbf{M}$ & $\mathrm{Y}$ & 72 & 44 & 85 & $22 \cdot 2$ & 3.4 & 48 & 58 \\
\hline 5 & 33 & 6 & $S$ & $\mathbf{N}$ & 61 & 46 & 71 & $10 \cdot 3$ & $1 \cdot 7$ & 46 & 96 \\
\hline 6 & 59 & 9 & $S$ & $\mathbf{N}$ & 66 & 35 & 66 & $68 \cdot 4$ & 32.5 & 238 & 159 \\
\hline 7 & 67 & 7 & $S$ & $\mathrm{Y}$ & 84 & 33 & 94 & $8 \cdot 8$ & $4 \cdot 7$ & 33 & 22 \\
\hline 8 & 69 & 7 & $\mathbf{M}$ & $\mathrm{Y}$ & 74 & 37 & 58 & $20 \cdot 7$ & $7 \cdot 3$ & 42 & 31 \\
\hline 9 & 66 & 6 & $\mathbf{M}$ & $\mathbf{N}$ & 81 & 39 & 76 & $18 \cdot 6$ & 6.9 & 62 & 74 \\
\hline 10 & 68 & 5 & $\mathbf{M}$ & $\mathbf{N}$ & 83 & 37 & 84 & $12 \cdot 0$ & 3.4 & 129 & 195 \\
\hline 11 & 58 & 9 & $\mathbf{M}$ & $\mathbf{Y}$ & 67 & 24 & 58 & $41 \cdot 0$ & $10 \cdot 2$ & 61 & 47 \\
\hline 12 & 60 & 7 & $\mathbf{M}$ & $\mathrm{Y}$ & 74 & 29 & 65 & $34 \cdot 1$ & $12 \cdot 4$ & 290 & 34 \\
\hline 13 & 66 & 5 & $\mathbf{M}$ & $\mathbf{N}$ & 76 & 40 & 92 & $8 \cdot 2$ & $4 \cdot 0$ & 34 & 27 \\
\hline 14 & 63 & 6 & $\mathbf{M}$ & $\mathbf{N}$ & 80 & 42 & 91 & 12.5 & $3 \cdot 1$ & 114 & 98 \\
\hline 15 & 60 & 5 & $\mathbf{M}$ & $\mathbf{N}$ & 77 & 36 & 98 & $8 \cdot 6$ & $3 \cdot 7$ & 52 & 21 \\
\hline $3^{\star}$ & 68 & 6 & $S$ & $\mathbf{N}$ & 74 & 39 & 73 & $10 \cdot 8$ & $3 \cdot 3$ & 42 & 33 \\
\hline $4^{\star}$ & 69 & 6 & $\mathbf{M}$ & $\mathbf{N}$ & 75 & 38 & 73 & 18.5 & 6.4 & 64 & 49 \\
\hline
\end{tabular}

Neoplastic nodules (S: single; M: multiple); ascites: presence $(\mathrm{Y})$ or absence $(N)$ of ascites; total protein: serum concentration of protein; albumin: serum concentration of albumin; PT: prothrombin time; total bilirubin: serum concentration of bilirubin; conjugated bilirubin: serum concentration of conjugated bilirubin

^Patients investigated again after 22 and 23 months respectively.

based on pertinent clinical and laboratory data and was confirmed by liver histology; according to the Child-Pugh classification, nine patients were in class A and eight in class B. HCC was diagnosed by liver sonography and cytological examination of ultrasound guided fine needle aspiration biopsy. One patient (no 14) had undergone segmental liver resection two years before admission. In six cases $\alpha$ fetoprotein values were over $200 \mu \mathrm{g} /$.

Using Okuda anatomo-clinical staging nine patients belonged to stage I, and eight to stage II. On examination by liver sonography, six cases showed a single neoplastic lesion and 11 had a multifocal HCC (five cases with two nodules, three with three nodules, and three with four or more nodules, respectively). Computed tomography after lipiodol administration showed multiple neoplastic foci in one patient with only one sonographically detected lesion. In the five cases with a single neoplastic lesion, neither liver resection nor percutaneous ethanol injection were feasible for anatomical reasons. Two patients (no 3 and no 4 ) had this treatment again because, after a successful TACE, a relapse was detected after 22 and 23 months, respectively. Data concerning this second treatment are included in the results.

The study was carried out in accordance with the declaration of Helsinki, and the protocol was approved by the relevant ethics authorities. Informed consent was obtained from all patients.

The Table reports clinical and baseline biochemical data of the patients.

\section{TACE procedure}

TACE was performed according to the method of Nakamura et al and was always preceded by coeliac, superior mesenteric, and hepatic arteriography, to evaluate the vascular anatomy of the liver and to exclude contraindications to TACE - that is, occlusion of portal vein or important arterovenous fistulas, or both. According to Seldinger's technique a catheter was inserted via the femoral artery in the proper hepatic artery or in a lobar or segmental branch feeding the tumour. As a rule, bilobar TACE was performed; in only five cases it was necessary to limit the procedure to a segmental or subsegmental TACE because of lowered portal blood flow, as assessed by portal angiography. An emulsion of $8.3(3.7)$ $\mathrm{ml}$ of Lipiodol (Laboratoire Guerbet, Aulnay sous Bois, France) and $40(15 \cdot 8) \mathrm{mg}$ of epirubicin (Farmorubicina, Carlo Erba-Farmitalia, Milano, Italy) was injected. Different doses were given in accordance with the tumour size. An embolising material composed of hand minced gel sponge (Gelfoam, Upjohn, Kalamazoo, Michigan, USA) suspended in diluted contrast medium was then injected. This injection was performed at small, repeated doses until lack of injection of the embolised vessels was obtained at angiography. Two weeks after the procedure computed tomography was done to demonstrate Lipiodol accumulation within the neoplastic lesions.

\section{Evaluation of metabolic activity of the liver}

Galactose elimination capacity - the day before TACE, after an overnight fast, galactose elimination capacity was performed by the single injection method. ${ }^{10}$ This method allows the estimation of the phosphorilating capacity of the liver, irrespective of any change in liver perfusion. Details of the procedure are described elsewhere. ${ }^{11}$ For each patient galactose elimination capacity in relation to body weight (galactose elimination capacity/ bw) was calculated. The test was repeated 24 hours and seven days after TACE using the same procedure in all the 17 cases.

Aminopyrine breath test - in six patients, five males and one female, aminopyrine breath test was measured before and seven days after performing TACE. This method ${ }^{12}$ evaluates the liver metabolic capacity of the P450 dependent enzyme system, ${ }^{13}$ and, because of the pharmacokinetic properties of the compound, the values obtained are not flow limited. Details of the procedure have been described elsewhere. ${ }^{11}$ 
Indocyanin green intrinsic hepatic clearance (ICG-IHC) - in 10 subjects a hepatic vein catheterisation was also performed, and ICGIHC was determined by the constant infusion method according to a previously described protocol ${ }^{14}$ before and 30 minutes after TACE.

\section{Evaluation of portal hypertension}

In the 10 patients undergoing hepatic vein catheterisation, hepatic venous pressure gradient was measured before and 30 minutes after TACE, as an index of portal hyper-
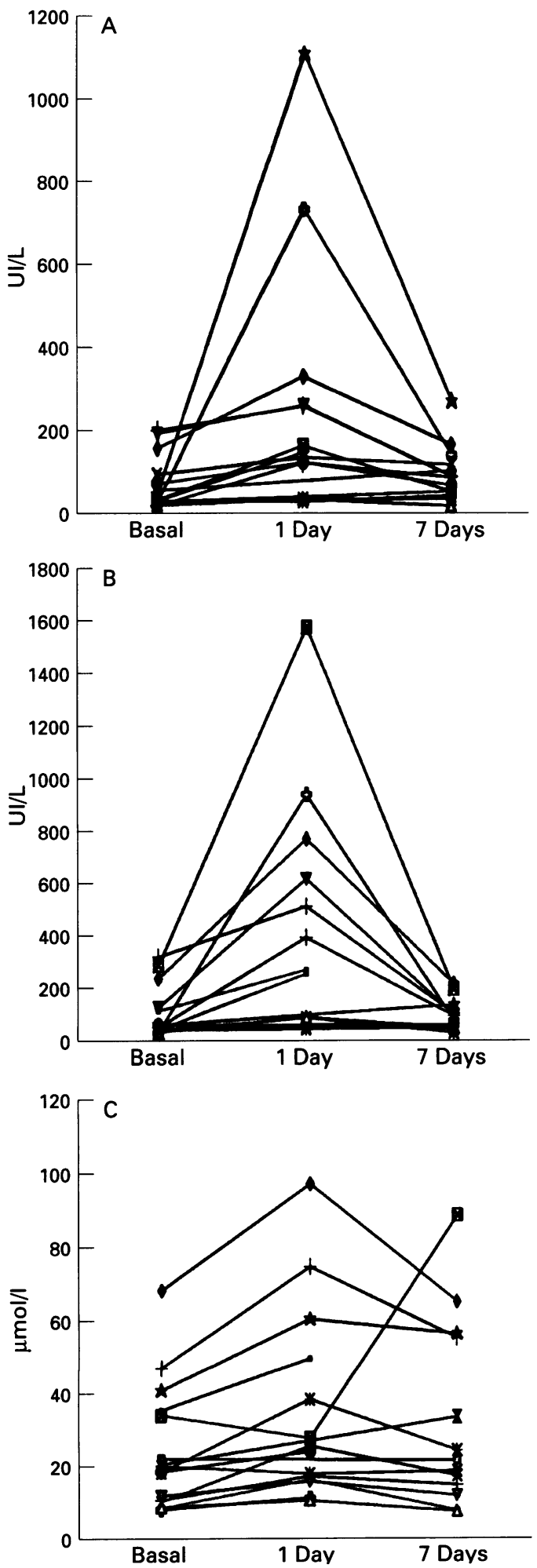

Figure 1: Changes in main biochemical data before and after TACE (( $A) A L T$, (B) $A S T$, (C) total bilirubin). tension. A balloon tipped catheter (Meditech 7 FR) was inserted in a main hepatic vein under fluoroscopic control, and free and wedged hepatic vein pressure were recorded by inflating the balloon until occlusion of the hepatic vein. Pressure was recorded with a pressure transducer, coupled to a monitor device (HP $1290 \mathrm{C}$ and HP 54S, Andover, Massachussetts, USA), and permanent traces were obtained. Electronic averages of pressure were always obtained. Adequate occlusion was assessed by injecting a small amount of contrast medium in the occluded vessel.

Statistics

All results are expressed as mean (SEM). The comparison between data was made using the Wilcoxon rank sum test. The null hypothesis was rejected at the 0.05 significance level. When the null hypothesis was not rejected, the power of the test was assessed according to standard statistical calculations.

\section{Results}

Changes in conventional tests and in clinical status

Significant changes in alanine transaminase, aspartate transaminase, serum bilirubin, and prothrombin values were observed 24 hours after TACE; all except serum bilirubin returned to baseline value within seven days (Fig 1). $\alpha$ Fetoprotein before TACE was 295 (103) $\mu \mathrm{g} / \mathrm{l}$. One month after treatment it decreased to $130(51) \mu \mathrm{g} / \mathrm{l}(\mathrm{p}<0.0001)$. The effect was particularly evident in patients with increased $\alpha$ fetoprotein values. Indeed, in the six patients with baseline $\alpha$ fetoprotein over $200 \mu \mathrm{g} / \mathrm{l}$, an average decrease by $42 \%$ was seen after one month.

No significant variation in clinical status of patients was seen one month after the procedure. No patient previously anascitic developed ascites and no case of portosystemic encephalopathy was seen. The Child-Pugh score was almost unchanged (before TACE $6.7(1 \cdot 3)$, after one month $6.8(1 \cdot 7)$, mean increase $1 \cdot 5 \%$ ).

\section{Effect on quantitative liver tests}

ICG-IHC, already considerably impaired before the procedure, showed a further decrease 30 minutes after TACE from 355 (140) $\mathrm{ml} / \mathrm{min}$ to 277 (98) $\mathrm{ml} / \mathrm{min}$, which averaged $22 \%(\mathrm{p}=0.0007)$ (Fig $2 \mathrm{~A})$.

Baseline galactose elimination capacity was $4.00(0.9) \mathrm{mg} / \mathrm{min} / \mathrm{kg}$ bw. One day after TACE no significant change was seen $(4 \cdot 20(0.9)$ $\mathrm{mg} / \mathrm{min} / \mathrm{kg} \mathrm{bw})(\mathrm{p}=0 \cdot 18$, power $88 \%)$. Seven days after TACE, values of galactose elimination capacity were not different from baseline $(3.95(0.87) \mathrm{mg} / \mathrm{min} / \mathrm{kg} \mathrm{bw})(\mathrm{p}=064$, power $96 \%$ ). Variations are given in Fig $2 \mathrm{~B}$.

When the 10 patients undergoing a more extensive procedure (bilobar TACE) were considered separately from those undergoing a more limited procedure (segmental or 

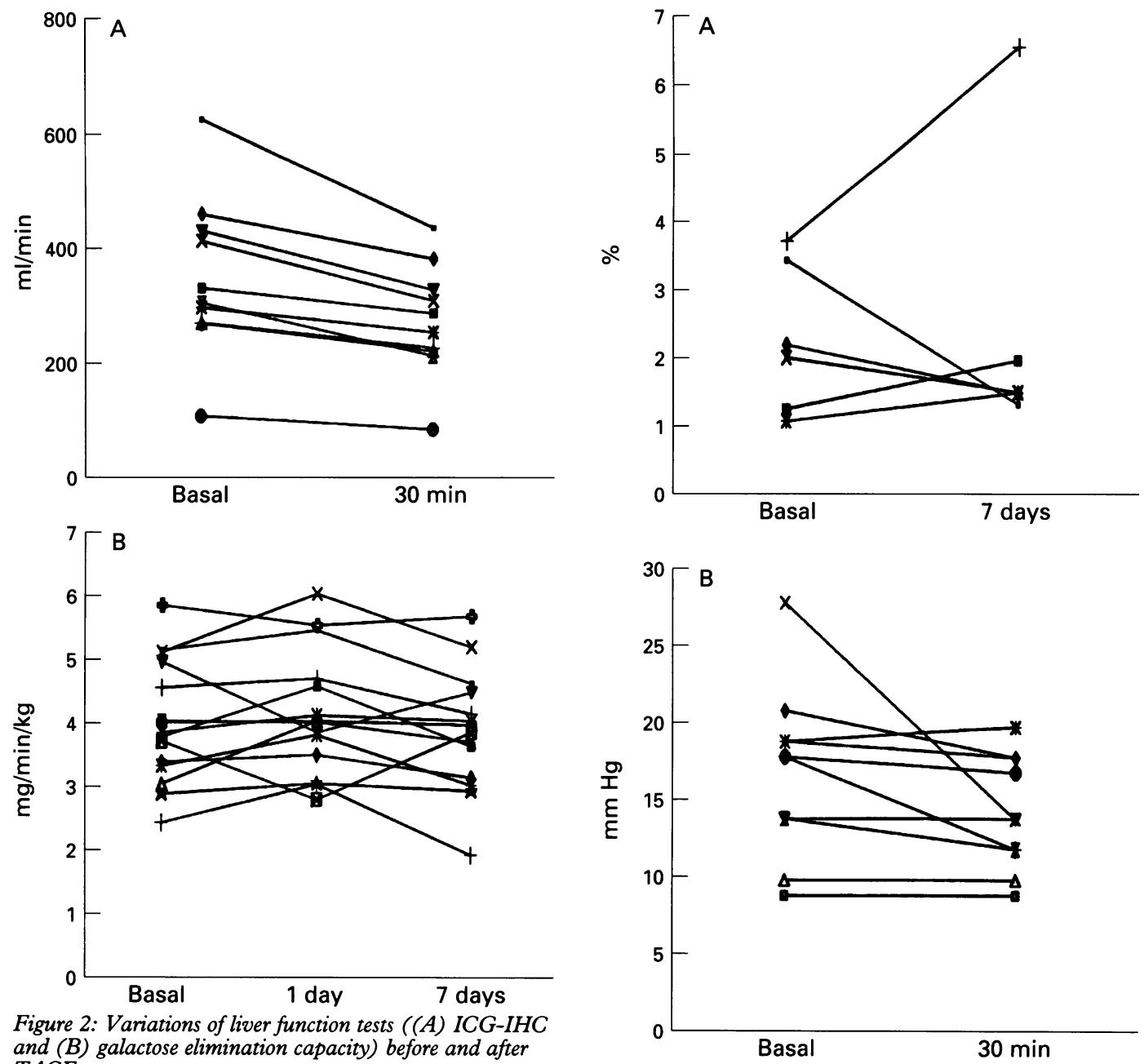

Figure 2: Variations of liver function tests $((A) I C G-I H C$ and $(B)$ galactose elimination capacity) before and after $T A C E$.

subsegmental TACE), no significant variation in galactose elimination capacity was seen. Indeed, in patients with bilobar TACE galactose elimination capacity changed from $4.02(0.93) \mathrm{mg} / \mathrm{min} / \mathrm{kg}$ bw to $4.30(0.99)$ $\mathrm{mg} / \mathrm{min} / \mathrm{kg}$ bw and to $4.02(0.93) \mathrm{mg} / \mathrm{min} / \mathrm{kg}$ bw $(p=0.066$, power $94 \%$; and 0.092 , power $98 \%$ respectively one and seven days after treatment). Baseline aminopyrine breath test was $2.31(1.09) \%$, and remained unchanged seven days after treatment: $2.39(2.04) \%$ $(\mathrm{p}=0.91$, power $95 \%)$ (Fig 3A).

Baseline HVPG was $17 \cdot 0(5 \cdot 5) \mathrm{mm} \mathrm{Hg}$ and 30 minutes after TACE it was $14.4(3.7) \mathrm{mm}$ $\mathrm{Hg}(\mathrm{p}=0.09$, power $99 \%)$. In particular, in the two patients with minimal portal hypertension, no change occurred, while in half of those with more severe portal hypertension a noticeable decrease was observed (Fig 3B).

\section{Discussion}

The results of this study show that the use of TACE to treat HCC in cirrhotic patients has only a transient effect on metabolic activity of the liver, as measured by three quantitative liver function tests, namely ICG-IHC, galactose elimination capacity, and aminopyrine breath test. Thirty minutes after the procedure, a distinct decrease in ICG-IHC was seen, averaging $22 \%$. This effect was less evident in patients with more severe liver

Figure 3: Modification in aminopyrine breath test $(A)$ and hepatic vein pressure gradient (B) before and after TACE.

function impairment. At variance, at one and seven days after TACE, galactose elimination capacity and aminopyrine breath test remained unchanged from baseline.

The decrease in ICG-IHC cannot be ascribed to the decrease in liver blood flow in itself, as intrinsic clearance is flow independent by its definition. ${ }^{15}$ On the contrary, it may result from a decrease in the number of hepatocytes, or a decrease in the function of the single hepatocyte, or the interruption of blood flow in discrete areas of the liver. Indeed, the intra-arterial infusion of iodised oil may provoke, at least transiently, an interruption of blood flow in some sinusoids, which are filled by the oily emulsion. ${ }^{16}$ This mechanism excludes such areas from blood exchanges, and consequently from the access to the dye, leading to a decrease of calculated intrinsic clearance. This may be responsible, at least in part, for the decrease in ICG-IHC seen in our patients, even if we cannot exclude that the reduction of function in single hepatocyte caused by the substances used may play a part in this phenomenon.

Galactose elimination capacity represents an estimate of the liver phosphorilating capacity for sugars, ${ }^{10}$ and aminopyrine breath test an estimate of the metabolic capacity of the P-450 dependent liver microsomal enzymes. ${ }^{13}$ Both techniques supply data independent of liver blood flow. The lack of decrease in galactose 
elimination capacity or aminopyrine breath test one and seven days after TACE implies that no consistent impairment in liver metabolic capacity occurred in our subjects at this time. These data are different from those reported in a Japanese study ${ }^{4}$ in which half of the patients experienced a decrease in maximum removal of ICG measured within four weeks after TACE. This discordance may result from the different selection of patients, because in that study some patients had HCC superimposed on liver disease other than cirrhosis. The data of our study also differ from those of an Italian study in which an increase in antipyrine clearance was seen. ${ }^{5}$ Induction of P-450 dependent enzymes by drugs may be responsible for this event, as it is not likely that TACE may have beneficial effects on liver function by itself.

The effect of TACE on the levels of sinusoidal portal hypertension has not been investigated. In this study, half of the patients with severe portal hypertension showed a pronounced decrease in HVPG, while those with mild and clinically unrelevant portal hypertension did not exhibit any change. The decrease in HVPG we observed may be caused by the decrease in arterial blood flow to the liver induced by embolisation because it was shown that the terminal arterial vasculature feeds the sinusoids directly ${ }^{17}$ or by a reflux of the dye towards the portal tree, which may impair the portal blood inflow to sinusoids ${ }^{16}$ or by a combination of both. In one of the three patients with severe portal hypertension and no change in HVPG, only a segmental TACE had been performed, and this may have led to only minor changes in liver haemodynamics.

This study cannot define the real clinical benefit of TACE because a randomised untreated group was unavailable.

However, the fall in $\alpha$ fetoprotein one month after the procedure in most subjects with increased baseline values may be considered a surrogate marker of effectiveness of treatment, ${ }^{18}$ considering that spontaneous decrease in $\alpha$ fetoprotein in HCC is an infrequent event. This decrease shows that the procedure had a biological effect on the tumour mass, and that the lack of effects on liver metabolic activity and on clinical status of patients is not caused by a lack of action on the neoplasm.

In conclusion, the results of this study show that TACE in cirrhotic patients with HCC provokes an acute decrease in ICG-IHC. At variance, no relevant variation in the other liver function tests, namely galactose elimination capacity and aminopyrine breath test, was seen one and seven days after treatment. Therefore a single TACE procedure seems to be a safe therapeutic tool for HCC in Child's class A and $\mathrm{B}$ cirrhotic patients.

This study was in part supported by grants from the Italian Ministry of University and Scientific Research (National Project 'Liver cirrhosis and virus hepatitis'), from the Italian National Council for Research (Project ACRO subproject 5), and from the Italian Liver Foundation, Florence.

1 Dusheiko GM, Hobbs KE, Dick R, Burroughs AK Treatment of small hepatocellular carcinomas. Lancet 1992; 340: $285-8$.

2 Okuda K, Okuda $\mathrm{H}$. Primary liver cell carcinoma. In: McIntyre N, Benhamou JP, Bircher J, Rizzetto M, Rodes , eds. Oxford Textbook of clinical hepatology. Oxford Oxford University Press, 1991: 1019-53.

3 Group d'Etude et de Traitement du Carcinome Hépatocellulaire. A comparison of Lipiodol chemoembolization and conservative treatment for unresectable hepatocellular carcinoma. $N$ Engl f Med 1995; 332: 1256-61.

4 Miyoshi S, Minami Y, Kawata S, Imai Y, Saitoh R, Noda S, et al. Changes in metabolic activity of the liver reserve after transcatheter embolization of hepatocellular carcinoma. $\mathcal{H}$ Hepatol 1988; 6: 332-6.

5 Grieco A, Marmiroli L, Marcoccia S, Castellano R Cotroneo A, Rosetto ME, et al. The functional hepatic reserve during the arterial lipiodol-carboplatinum therapy in cirrhotic patients and hepatoma. Ital f Gastroenterol 1993; 25 (suppl 1): 98.

6 Moriyasu F, Ban N, Nishida O, Nakamura T, Soh Y, Miura $\mathrm{K}$, et al. Portal hemodynamics in patients with hepatocellular carcinoma. Radiology 1986; 161: 707-11.

7 Taourel P, Dauzat M, Lafortune M, Pradel J, Rossi M, Bruel JM. Hemodynamic changes after transcatheter arterial embolization of hepatocellular carcinoma. Radiology 1994; 191: 189-92.

8 Okuda K, Ohtsuki T, Obata H, Tomimatsu M, Okazaki N, Hasegawa $\mathrm{H}$, et al. Natural history of hepatocellular Hasegawa $\mathbf{H}$, et al. Natural history of hepatocellular carcinoma and prognosis in relation to treat

9 Nakamura H, Hashimoto T, Ohi H. Transcatheter oily chemoembolization of hepatocellular carcinoma. Radiology 1989; 170: 783-6.

10 Tygstrup N. Determination of the hepatic elimination capacity $(\mathrm{Lm})$ of galactose by single injection. Scand $\mathcal{F}$ Clin Lab Invest 1966; 18 (suppl 92): 118-25.

11 Merkel C, Sacerdoti D, Finucci GF, Bazzerla G, Bolognesi M, Gatta A. Effect of nadolol on liver hemodynamics and function in patients with cirrhosis. Br 7 Clin Pharmacol 1986; 21: 713-9.

12 Hepner GW, Vesell ES Quantitative assessment of metabolic activity of the liver by breath analysis after oral administration of 14-C-aminopyrine. Ann Intern Med 1975; 83: 632-8.

13 Brodie BB, Axelrod J. The fate of aminopyrine (pyramidon) in man and methods for the estimation of aminopyrine and its metabolizing in biological material. $\mathcal{f}$ Pharmacol Exp Ther 1950; 99: 171-84.

14 Merkel C, Bianco S, Bolognesi M, Caregaro L, Amodio P, Sacerdoti D, et al. Effect of triglycyl-lysin-vasopressin on quantitative liver function tests in patients with cirrhosis. Am $\mathcal{F}$ Gastroenterol 1992; 87: 1580-6.

15 Bass L, Keiding S, Winkler K, Tygstrup N. Enzymatic elimination of substrates flowing through the intact liver. Theor Biol 1976; 61: 393-410.

16 Kan $Z$, Sato $M$, Ivancev $K$, Uchida $B$, Hedgpeth $P$ Lunderquist A, et al. Distribution and effect of iodized poppyseed oil in the liver after hepatic artery embolization: experimental study in several animal species. Radiology 1993; 186: 861-6.

17 Watanabe Y, Püschel P, Gardemann A, Jungermann K Presinusoidal and proximal intrasinusoidal confluence of hepatic artery and portal vein in rat liver: functional evidence by orthograde and retrograde bivascular perfusion. Hepatology 1994; 19: 1198-207.

18 Mondazzi L, Bottelli R, Brambilla G, Rampoldi A Rezakovic I, Zavaglia C, et al. Transarterial oily chemoembolization for the treatment of hepatocellular chemoembolization for the treatment of hepatocellular Hepatology 1994; 19: 1115-23. 